\title{
Construcción de las identidades latinoamericanas. Una aproximación desde el entorno sonoro
}

\author{
Constructing Latin American Identities. \\ An Approach from the Sound Environment Perspective
}

Construção de identidades latino-americanos. Uma abordagem desde o paisagem sonoro

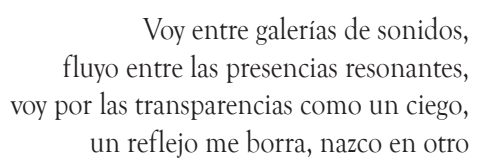

Octavio Paz

Francisco Rodríguez-Varela

Maestría en Estudios Latinoamericanos

Universidad Nacional de Costa Rica

Recibido: 9 de mayo 2016

Aceptado: 10 de julio de 2016

\section{Resumen}

Este acercamiento pretende problematizar la dimensión sonora como uno de los elementos estructurantes de las identidades, tanto individuales como colectivas, en un contexto determinado, permitiendo así, establecer un puente

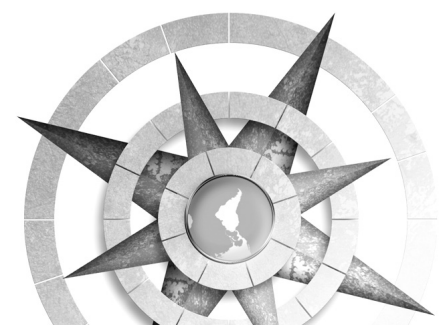

epistémico entre las estructuras hegemónicas propias del colonialismo y su intrínseca relación con el paisaje sonoro latinoamericano. Se busca visibilizar cómo han perdurado y mutado en la actualidad las tendencias de pensamiento colonialistas, prestando especial importancia al sonido como una unidad de análisis que necesariamente acompaña a dichas estructuras de pensamiento en cada una de las dimensiones en las 
que se desenvuelve el ser humano: económicas, políticas, culturales, artísticas, tecnológicas, recreativas, entre otras.

Palabras clave: Identidades, estructuras ideológicas, pensamiento colonialista, paisaje sonoro, epistemología, América Latina

\begin{abstract}
This approach intends to problematize the sound dimension as one of the structuring elements of identities, both individual and collective ones, in a specific context; thus, it is possible to establish an epistemological bridge between hegemonic structures of colonialism and its intrinsic relation with the Latin American sound landscape. The aim is to make visible how the current colonialist trends of thought have remained and transformed. Special importance is given to the sound as a unit of analysis that necessarily accompanies such structures of thought in each one of the dimensions in which human being lives: political, cultural, artistic, technological, recreational ones, among others.
\end{abstract}

Keywords: Identities, ideological structures, colonialist thought, sound landscape, epistemology, Latin America

\section{Resumo}

Este abordagem visa problematizar dimensão sonora como um dos elementos estruturais de identidades, individuais e colectivas, em um determinado contexto. Procura estabelecer a ponte epistémico entre estruturas próprias hegemónicas do colonialismo e sua relação intrínseca com paisagem sonora da América Latina. Neste ensaio visibiliza como as atuais tendências colonialistas do pensamento sofreram e se transformaram com particular destaque para o som como unidade de análise que acompanha essas estruturas do pensamento.

Palavras chave: Identidades, estruturas ideológicas, pensamento colonialista, paisagem sonora, epistemologia, América Latina

Detenernos a escuchar los diversos sonidos que confluyen a nuestro alrededor, implica poner nuestra atención sobre la historia. Efectivamente, esta invitación a de-construir nociones que naturalizan los registros sonoros como elementos neutros, ahistóricos, abstractos y ajenos a los procesos socio-culturales, necesariamente dificulta la elaboración de análisis rigurosos que permitan reconocer narratividades en los "paisajes sonoros" (Barrios y Ruiz, 2014, p.57.), es decir, identificar cómo a través del 
tiempo existen sonidos que expresan las particularidades de contextos en los cuales nos socializamos. Al respecto Barrios y Ruiz (2014) comentan que:

Estamos inmersos en sonidos, los cuales provienen de distintas fuentes con sus diversas tonalidades y volúmenes, que se unen y se mezclan entre ellos haciendo que un lugar suene de manera peculiar y diferente a cualquier otro... A todo ese entorno de sonidos que percibimos se le llama "paisaje sonoro" (p.57).

A este respecto, hemos de identificar uno de los hechos relevantes entre los paisajes sonoros y los espacios en los cuales se socializan las personas y que tiene que ver con la construcción de las identidades en el contexto latinoamericano. De aquí la importancia de plantear una discusión teórico-metodológica que problematice las circunstancias socio-históricas en las cuales las identidades latinoamericanas comienzan a tomar un nuevo viraje en relación a la época colonial y posteriormente con la modernidad.

La identidad como categoría de análisis se ha convertido para los sectores académicos en una obsesión: ¿Quiénes somos los latinoamericanos? ¿Cómo nos definimos frente a un otro? o mejor dicho ¿Cómo queremos ser definidos frente a un otro? ¿Qué nos caracteriza como la- tinoamericanos? Claro está que la constante necesidad de poder definirnos, de poder situarnos en el mundo, responde a una necesidad ontológica. ¡Por supuesto! No es casualidad que las continuas interrogantes sobre la identidad que nos planteamos sean de corte filosófico; así, la historia nos da un trazo de huellas que nos sitúa en una encrucijada: la época colonial, período que podríamos caracterizarlo como un tiempo intenso, por una parte por los cambios socio-culturales, económicos e infraestructurales que vivieron los pueblos originarios, así como también por las formas tan violentas en que fueron impuestas las prácticas ideológicas del colonizador europeo.

La forma de acercarnos a la reflexión sobre las identidades en este ensayo, implica reconocer al menos en un primer nivel de discusión, una doble ruta sobre la que opera esta categoría, pues a partir de la época colonial no solo se instaura en la psique latinoamericana una obsesión ${ }^{47}$ por tener clara cuál es nuestra posición en el mundo, sino que a la vez,

47 Se entiende el término obsesión a partir de Rodríguez de Castro (2004): "El fenómeno obsesivo en general, se sitúa exactamente entre el control y la pérdida de control y expresa la tensión existente, la tirantez, entre los dos polos." (p.6) con lo cual, la idea de obsesión permite anclar un extremo de pérdida identitaria como resultado de los procesos de colonización y, por otra parte, los considerables esfuerzos teóricos por entender los rasgos que caracterizan las identidades latinoamericanas para así recuperar simbólicamente, el control sobre nuestra existencia. 
nos pone en un plano de ordenamiento existencial en el que, la diversidad cultural queda homogeneizada a través de la invención del sujeto indio, firmemente legitimado a través de la raza.

La idea de raza en nuestras culturas nace precisamente de la semilla colonial, que impositivamente nos situó en un lugar del espacio físico y simbólico -cabe mencionar, de inferioridad y sumisión- a partir del cual empezamos a dar nuestros primeros pasos para conformar nuestras propias identidades. Es por ello que se puede comprender en cierta medida cómo nuestras identidades latinoamericanas están en contra, o al menos en desacuerdo con el movimiento que nos engendró.

Ahora bien, se debe hacer hincapié sobre lo trascendental que significó la instauración de una mentalidad colonial en el imaginario social latinoamericano, ya que este viraje cultural y epistemológico (por los condicionamientos a las diversas formas de pensar (nos) nuestro entorno) también ocurre en un cambio radicalmente cualitativo del entorno sonoro de nuestros pueblos originarios. El disciplinamiento del oído para acostumbrarnos a nuevos sonidos, forman parte de una gran orquesta estructural que trastoca todas las dimensiones y sentidos del ser humano. Por ahora basta mencionar cómo esta mentalidad colonial trajo consigo una imitación acrítica conductual hacia el colonizador: ser como ellos se tornó el norte de nuestro propósito existencial. Asimismo el predominio del centro metropolitano como modelo de humanidad queda legitimado en la nueva configuración socio-espacial en nuestros territorios.

El proceso de colonización introdujo en la psique humana las nociones de centro-periferia, de esta forma el complejo de superioridad europeo sin lugar a dudas encontró como uno de sus nichos la asociación político-ideológica: periferia/ subdesarrollo-centro/desarrollo. A su vez el cuerpo europeo re-aparece en escena como el héroe, que teniendo la Biblia como instrumento, justifica la evangelización de nuestros antepasados, lo que incluye a su vez, dar muerte a la compleja construcción de diversas formas de pensamiento. Claro está que, si bien la religión jugó un papel trascendental en todo el proceso de la conquista, será la razón científica la protagonista por excelencia que se autoproclamará en los imaginarios sociales. Producto de esto, el horizonte ontológico trazado ancla al cuerpo europeo y a la razón científica como el conditio_sine qua non, esto es, componentes sin los cuales no es posible pensar la historia latinoamericana; precisamente esta característica sirve de caldo de cultivo para la modernidad, que funda su proyecto de la conquista justificada en 
el conocimiento avanzado del europeo versus el bárbaro indígena.

La polémica es evidente, el gran ojo observador es el equivalente a un sujeto óntico, al respecto Aimé Cesaire (2006) señala que: "La «ego-politica del conocimiento» inaugurada con René Descartes en el siglo XVII inicia el mito del sujeto que piensa desde «el ojo de Dios»." (p.152). Se trata de aquel sujeto que conoce y que controla, que construye el mundo a partir de su lógica de entendimiento, sin embargo, como locus de enunciación se presenta desde un no lugar (Augé, s.f., p. 3) neutro, objetivo y omnipresente.

Se trata así de enfocarnos en el lenguaje, específicamente en el poder del discurso social, pues como explica Bofill (1943), la misma palabra autor (párr. 5) tiene un estrecho ligamen con la palabra autoridad (párr. 5), en consecuencia, el mundo queda sujeto y supeditado a la voz activa y autor-itaria de quien construye, delimita, permite, señala y castiga entre otros. Es un juego de construcción de identidades, de vigilancia y castigo respecto de otros discursos o visiones de mundo, pues lo que se emite/ omite, resalta/oculta y domina/somete tiene que ver con el locus de enunciación respecto de cómo deberían ser las cosas, por lo que se excluye a la vez, todo aquello que no concuerde con la lógica de la voz autoritaria.
Ahora bien, la sutileza con la que han operado las numerosas formas de colonización en nuestro continente, nos obliga a cuestionarnos sobre las diversas maneras tan sutiles de colonialismos que han perdurado en nuestra realidad y que tienen una influencia directa sobre la construcción de las identidades latinoamericanas.

Efectivamente la palabra sutil se utiliza para dar cuenta de la sospecha epistemológica que como profesionales debemos aplicar en todos los ámbitos de la vida cotidiana, de forma tal que se des-naturalicen procesos que surgen como producto de una compleja construcción social, o dicho en otros términos, que no responden al orden de lo biológico.

De esta forma cabe preguntarnos: ¿Qué relación existe entre el sonido y el conocimiento? ¿Cuáles son algunas de las influencias que tienen los entornos sonoros sobre las identidades de los colectivos sociales? O bien ¿Qué puentes epistemológicos se pueden establecer entre el sonido y toda la influencia de la época colonial en nuestra región latinoamericana? Tracemos unas primeras líneas de discusión.

Sin lugar a dudas las experiencias sonoras de nuestros pueblos originarios tenían matices que no pueden ser com- 
parables con los sonidos que experimentamos en nuestros días. En primera instancia podemos afirmar que la relación intrínseca que existía entre los elementos naturales y los rituales de los indígenas daba cuenta de una estructura sonora muy particular; así, el trueno, el fuego, el viento, el agua, la tierra, entre otros, conformaban no solo un paisaje visual que permitía situar ontológicamente a los indígenas en el mundo sino que a la vez conformaban un paisaje sonoro que les permitía identificarse con el entorno. ¡Claro! Imaginar a nuestros antepasados sin el elemento sonoro es el equivalente a construir una película mental muda en la que los indígenas se socializan en un mundo sin sonido.

Como consecuencia, ontología y sonido dialogan en el devenir de la historia y desde el punto de vista funcional-estructural, el sonido nos permite identificarnos con el entorno. A su vez las experiencias sonoras dan sentido a nuestra existencia ya que posibilitan contextualizarnos y situarnos en el mundo ¿o acaso al levantarnos escuchamos sirenas de la Segunda Guerra Mundial alertándonos ataques aéreos? ¿Caminamos por la calle escuchando el sonido de una AK47 o el grito de una joven iraquí siendo violada por diez militares estadounidenses?
La respuesta es obvia: la estructura de sonidos que forman parte de nuestro entorno dan cuenta del contexto en el que vivimos, su existencia en la cotidianeidad nos da la seguridad de que estamos en el realidad correcta, en el escenario pertinente o lógico. Imaginar despertar en un paisaje de sonidos radicalmente distintos a los que estamos acostumbrados supondría pensar en la locura o el suicidio como posibilidades ante una situación tan angustiante.

La importancia de los elementos naturales en la estructura cultural de nuestros antepasados, fue mayúscula. Podemos decir que muchos de estos componentes del orden de lo natural simbolizaban figuras idolatradas, totémicas, esto es, en una relación espiritual construida por los indígenas. Se trata en determinados casos de dioses que tomaban la forma de un rayo, por ejemplo, o de cualquier otra manifestación de la naturaleza. Es en este punto donde debemos hacer consciencia y visibilizar un punto objetivo: los dioses de nuestros antepasados tenían una voz; no eran figuras áfonas, silentes, sus diversas formas de manifestación dentro del sistema de saberes de los indígenas pasaban por un registro sonoro que daba cuenta de su poder, de su mandato en el mundo de lo terrenal. 
Lo anteriormente expuesto reviste de gran importancia en el mundo académico: se trata de dar cuenta de una estructura óntica en extremo compleja, que da como resultado identidades específicas en el mundo, para este caso particular, pueblos originarios que se relacionan de formas singulares con su entorno. Y sobre todo porque existe un punto de quiebre en la psique de nuestros pueblos originarios: la conquista. La época colonial para Maldonado-Torres (2007) implica un proceso de mutilación, apropiación de las tierras, racialización y esclavismo como las principales prácticas de la colonialidad, de esta forma como bien explica el autor, aparece el ego conquiro (p. 133) como la noción que permite dar paso al ego cogito cartesiano. Se trata del nacimiento de una relación epistemológica fundada en los epistemicidios (de Sousa, 2010, p. 8) en el cual el sujeto es y se constituye en la acción de conquistar, es decir, yo conquisto-luego existo. La principal implicación de esta lógica tiene como referente el no re-conocimiento a la cosmovisión de nuestros antepasados.

En este mismo sentido, Moreno Fraginals (2000) identifica en la colonización procesos de deculturación en la conformación identitaria de nuestros pueblos, en los cuales, por fines de explotación económica, se sustentan desarraigos culturales para facilitar la expropiación de riquezas. La deculturación tiene ba- ses objetivas: la puesta en escena de los pueblos indígenas en el sistema laboral internacional, la construcción de un imaginario dicotómico entre el nosotros y los otros, las lecciones humanísticas dadas por Ginés de Sepúlveda y descritas por Lipshutz (1975), entre otros ejemplos.

Pero cabe preguntarse qué consecuencia tuvo la colonización en los registros sonoros de nuestros pueblos. La respuesta no es única, ni reduccionista. Supone, en primera instancia, poder examinar el cambio cualitativo de la relación de los indígenas con la naturaleza y sus deidades o figuras divinas. Así pues, Sepúlveda en su Tratado sobre las justas causas de la guerra contra los indios (en Lischutz, 1975) se refiere a nuestros antepasados en los siguientes términos:

...esos hombrecillos en los que apenas encontrarás vestigios de humanidad; que no sólo no poseen ciencia alguna, sino que ni siquiera conocen o usan las letras ni conservan ningún monumento de su historia sino cierta obscura y vaga reminiscencia de algunos hechos consignados en ciertas pinturas, y tampoco tienen leyes escritas, sino instituciones y costumbres bárbaras (p. 72)

Sepulveda, entre otros, nos enseñó el camino verdadero, el del Dios cristiano, aquel que representa el pasaje derecho por el cual debían transitar los indíge- 
nas. Algunos de los cuestionamientos que salen a la luz, prosiguiendo en este ámbito de lo divino, en este hito histórico son: ¿Cómo sonaba ese Dios cristiano? ¿Qué registro visual y sonoro daba cuenta de su existencia? ¿Una vez que el indígena aprendió el castellano, qué pasó por su mente cuando se le dijo que el nuevo Dios era una figura omnipresente y de fe, pero no tenía referente existencial-empírico en lo terrenal?

Si de algo estaban seguros los indígenas es que esta nueva figura a la cual debían rendir adoración era un Dios mudo: ¡sí, mudo! en el estricto sentido, pues no existía registro sonoro en lo terrenal que pudiera dar cuenta de su existencia lo cual estructura la psique de manera radicalmente distinta en comparación con los diálogos establecidos anteriormente entre los indígenas y sus figuras de adoración en la naturaleza. Esto también cambiaría las interpretaciones a los sonidos del entorno natural, las cuales estarían más ligadas al filtro de la razón científica como columna vertebral en la nueva re-lectura al mundo.

En el plano epistémico, la época colonial nos obligó a educar nuestro oído a acostumbrarnos a sonidos civilizatorios, es decir, hubo una transformación en el tanto que se nos exigió, violentamente, una nueva forma de escuchar, legitimando entornos sonoros que no eran pro- pios de nuestras culturas y escuchando sonidos que son el producto de una violación simbólica.

Algunos de ellos son la voz autoritaria del hombre blanco dando la orden de saqueamiento y asesinato, los gritos de miles de mujeres indígenas siendo violadas por los conquistadores, la espada que desenvaina el español y entra en el cuerpo del indígena, los caballos que galopan y dejan consigo una estela de muerte, las herramientas hechas de hierro utilizadas en diversas labores, el sonido de las cadenas para aprisionar al indígena, el sonido de los utensilios no solo en el trabajo de labranza sino también en la propia cocina, las campanas que dan cuenta de un ritual religioso, las ondas de la voz y de todos los sonidos que ya no chocan en un ambiente predominantemente de naturaleza, sino por el contrario, en un entorno de mampostería, el látigo, el cañón, las pistolas y muchos otros; todos ellos suponen la entrada a nuestra nueva fuente sonora. Serán esos sonidos con los que se asociará la evolución del indígena para ser reconocido como digno de la raza humana -aunque claramente en una posición de desventaja-, exorcizado de su barbarie y constituido en el plano ontológico como un ser, esto es, como alguien que ahora cobra alguna importancia para el occidental en el plano existencial. 
Indiscutiblemente el crimen realizado tuvo que ver, en gran medida, con una educación en la que nuestro cerebro procesó entornos sonoros extremadamente violentos, cuyos sonidos, según se nos dijo, eran sonidos de civilización. De esta forma el temperamento sonoro en Latinoamérica - proveniente directamente de la alta alcurnia española- era simplemente eso: una sentina de sonidos sin precedente alguno en nuestra región.

Desde una lectura teórica, podemos identificar cómo estos nuevos sonidos de trabajos no comunes, de tonalidades de voz del español, de nuevos dialectos al aire, producto de la traída de africanos, de herramientas y animales, entre otros, en un primer momento generaron un caos existencial, una des-localización. Sin embargo, lamentablemente fuimos forzados a legitimar este entorno sonoro como parte de nuestra identidad latinoamericana, a naturalizar el ruido del metal, del hierro, del trajín acelerado de la metrópoli como parte de nuestras vidas, lo que necesariamente nos ha llevado tener, como sello característico, una constante conflictividad existencial y una actitud pasiva frente a paisajes sonoros que surgen como producto de la violencia.

Ahora bien, posterior al período colonial interesa identificar un hecho de especial importancia, vinculado a la épo- ca independentista y la conformación de los Estados-Nación, pues si bien no se modifican significativamente las estructuras económicas y socio-coloniales, es real desde el campo administrativo, una emancipación política de parte de nuestros pueblos. Es decir, la Corona Española se desplaza, en parte porque no permitía un desarrollo productivo propio de las periferias. Consecuentemente el gran proyecto nuevo empujará por la modernización del Estado y en una disputa constante con el sector clerical por la tenencia de tierra.

Asimismo, los nacionalismos son fuertemente trabajados desde la dimensión simbólica, la construcción social de monumentos, héroes nacionales, canciones y comidas populares, bulevares, edificios entre otros, se suman a esta intención de modernización, permitiendo con ello generar procesos de homogeneidad cultural o, entendido en otros términos, la lógica imperante se basaba en la relación, yo homogeneizo/luego existo.

Pero también la modernidad supuso un impacto directo sobre el paisaje sonoro, en el tanto que, los sonidos homogeneizadores tenían su expresión en la gran obra industrial-mercantilista, aspecto que no solo conformó un avasallamiento sobre la voz humana y los sonidos de la naturaleza, sino que a la vez implicó que todos los sonidos provenientes de 
las grandes maquilas, las industrias manufactureras, así como también de las propias mercancías que en se producían en la metrópoli, ahora fueran lo más alabado de la época moderna.

Puesto en otros términos, las mercancías ahora se presentan como la gran salvación que llena los vacíos existenciales del ser humano, son los objetos materiales los encargados de civilizar y mejorar la calidad de vida de las personas.

Detengámonos brevemente en este aspecto, ¿permiten las mercancías la satisfacción de necesidades reales en el humano y nos facilitan la vida en la realización de nuestras tareas diarias? Desde el punto de vista de un paisaje sonoro en el que al mismo tiempo suena el timbre del celular e ingresa una llamada; la lavadora dispara una señal de que terminó el proceso de lavado, desde el televisor se nos informa la cantidad de muertos el día de hoy, la refrigeradora suena para indicar el aumento el enfriamiento; el grifo está abierto, el equipo de sonido se mezcla con las noticias del televisor; la impresora alerta de que se ha terminado la impresión; la contestadora toma un mensaje....

Este tráfico caótico de sonidos confluye en nuestra cotidianeidad. ¡Y solo refiere al espacio doméstico! Se trata de una estructura sonora que da cuenta de los nuevos colonialismos sonoros que van construyendo nuestras identidades, representan sonidos con los cuales nos identificamos, los hacemos nuestros, forman parte de nuestro sentido de vida en el mundo. Es por ello que compartimos una escucha latinoamericana, tenemos un oído propio, con una memoria histórica específica, pero adormecida, precisamente por la naturalización a dichos registros sonoros.

En síntesis, lejos de presentar un balance de resultados o conclusiones esta reflexión busca acortar la distancia en el plano epistémico entre la época colonial, las nuevas formas de colonialismo y los registros sonoros como un espectro o columna vertebral que permite dar cuenta de las estructuras de prácticas civilizatorias de occidente.

Es claro que se trata de un primer acercamiento epistemológico, que afortunadamente nos deja futuras discusiones abiertas donde necesariamente deberán opinar la mayor cantidad de disciplinas científicas con el fin de poder sumar esfuerzos a este tipo de análisis tan descuidados principalmente por las Ciencias Sociales y que buscan, no solo la comprensión rigurosa de fenómenos que nos interpelan, sino que a su vez suma luchas por la emancipación 
de una región que ha sido brutalmente violentada en todas sus dimensiones de pensamiento y acción.

\section{Referencias bibliográficas}

Augé, Marc (s.f.) Sobremodernidad. Del mundo de hoy al mundo de mañana. Consultado de: https://asodea. files.wordpress.com/2009/09/ auge-marc-sobremodernidad.pdf

Barrios, G.; Ruiz, C. El paisaje sonoro y sus elementos. Revista Quehacer Científico en Chiapas, 9(2). Consultado de: http://www. dgip.unach.mx/images/pdf-REVISTA-QUEHACERCIENTIFICO/QUEHACER-CIENTIFICO-2014-jul-dic/El_paisaje_sonoro_y_sus_elementos.pdf

Bofill, J. (1943) Autoridad, Jerarquia, Individuo. Revista de Filosofía. Año II, no 5, páginas 363-375.Madrid, abril-julio. Consultado de: http://www.filosofia.org/hem/ dep/rdf/005p363.htm

Cesaire, A. (2006). El discurso sobre el colonialismo. Editorial Akal S.A. Madrid España. Consultado de: http://www.alca-seltzer.org/descolonizacion/cesaire_libro_discurso_sobre_colonialismo.pdf

De Sousa, B. (2010). Decolonizar el Saber, Reiventar el Poder. Ediciones Trilce, Montevideo, Uruguay. Consultado de: http://www. boaventuradesousasantos.pt/
media/Descolonizar \%20el \%20 saber_final \%20- \%20C \%C3 \%B3pia.pdf

Lipschutz, A. (1975). El mundo indiano, visto por los contemporáneos de la conquista. El problema racial en la conquista de América. México: Editorial Siglo XXI. pp. 69-139

Maldonado-Torres, N. (2007). Sobre la colonialidad del ser: contribuciones al desarrollo de un concepto. En: El giro decolonial. Reflexiones para una diversidad epistémica más allá del capitalismo global. Colombia: Siglo Hombre Editores.

Moreno, Manuel (2000). Aportes culturales y deculturación. En África en América Latina México: UNESCO/Siglo XXI, pp. 13-33

Rodríguez de Castro, Luisa. (2004) La obsesión y el par antitético control-pérdida de control.. Consultado de: http://www.micropsicoanalisis.com/pdf/obsesion.pdf 